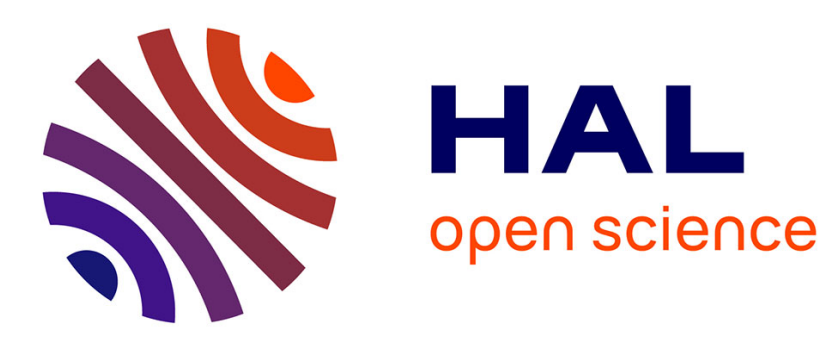

\title{
Spline approximation of gradient fields: applications to wind velocity fields
}

Carole Le Guyader, Dominique Apprato, Christian Gout

\section{To cite this version:}

Carole Le Guyader, Dominique Apprato, Christian Gout. Spline approximation of gradient fields: applications to wind velocity fields. Mathematics and Computers in Simulation, 2014, 97, pp.260279. 10.1016/j.matcom.2013.10.003 . hal-01022817

\section{HAL Id: hal-01022817 https://hal.science/hal-01022817}

Submitted on 10 Jul 2014

HAL is a multi-disciplinary open access archive for the deposit and dissemination of scientific research documents, whether they are published or not. The documents may come from teaching and research institutions in France or abroad, or from public or private research centers.
L'archive ouverte pluridisciplinaire HAL, est destinée au dépôt et à la diffusion de documents scientifiques de niveau recherche, publiés ou non, émanant des établissements d'enseignement et de recherche français ou étrangers, des laboratoires publics ou privés. 


\title{
Spline approximation of gradient fields: applications to wind velocity fields
}

\author{
Carole Le Guyader ${ }^{1}$ Dominique Apprato $^{2}$ and Christian Gout ${ }^{1}$ \\ 1: INSA de Rouen, Laboratoire de Mathématiques de l'INSA, Avenue de l'Universite, 76801 St Etienne du \\ Rouvray cedex France \\ 2: Université de Pau, LMA-CNRS, UFR Sciences et Technologies de la Côte Basque, All. Parc Montaury, 64600 \\ Anglet, France.
}

\begin{abstract}
We study a spline-based approximation of vector fields in the conservative case. This problem appears for instance when approximating current or wind velocity fields, the data deriving in those cases from a potential (pressure for the wind, etc..). In the modelling, we introduce a minimization problem on an Hilbert space for which the existence and uniqueness of the solution are provided. A convergence result in the introduced Sobolev space is established using norm equivalence and compactness arguments, as well as an approximation error estimate of the involved smoothing $D^{m}$-splines.
\end{abstract}

Keywords:

Approximation, smoothing problems, vectorial splines, convergence, Sobolev space, conservative vector fields.

AMS subject classification: 41A15, 41A25, 68U10.

\section{Introduction}

The problem of vector field approximation emerges in a wide range of fields such as: motion control, computer vision, geometrical analysis, geometrical design, analysis of acoustic and/or electromagnetic waves, as well as in geophysics, medical imaging, fluid mechanics and so on... Many different approaches have been introduced to solve each specific problem occuring in the above fields of investigation. One can mention for instance: finite element approximation (see Dzhabrailov et al. [14]), PDE-based methods (see Amodei and Benbourhim [1]), spline and Rational Basis Function approximations (see Awanou and Lai [6], Dodu and Rabut [11], Benbourhim and Bouhamidi [7]-[8], Ettl-Lowitsch et al. [15, 17, 18], Zhou et al. [25]...).

The originality of this work consists:

- in considering that the vector field derives from a potential (conservative vector field): it occurs for instance in meteorology (winds derive from temperature potentials), oceanography (currents derive from pression potentials), image processing... 
- of a rigorous study of existence-uniqueness of the solution of the problem phrased as an energy minimization,

- in establishing a convergence result (while many approaches only give algorithms without mathematical study) and providing an approximation error estimate.

From the best of our knowledge, our approach is the first one of this kind. Moreover, we propose a method easy to implement and parallelize, using classical tools like the least-square method and the finite element method (for the discretization).

More precisely, in this work, we do not want to compute a potential that could generate the vector field data. We only want to get a global approximation of the vector field dataset on a bounded domain, taking into account in the modelling that this approximation derives from a potential. Furthermore, contrary to interpolation methods, we prefer to fit the vector field dataset in the case of realistic data (when the number of vectors is large or when the data are corrupted by noise). To achieve this, we introduce a minimization problem defined as a regularized leastsquare problem formulated on a Sobolev space of potentials (see subsection 2.1). Obviously, this problem has an infinite number of solutions, but we derive from it a problem expressed in terms of the gradient vectors. We prove that the associated problem in terms of vectors has a unique solution which is the corresponding approximation of the vector field dataset (subsection 2.2). In subsection 2.3, we give a convergence result when the number of vectors increases to infinity. In Section 3, we give the discretization complemented by an approximation error estimate of the involved smoothing $D^{m}$-splines. We then give numerical results in Section 4 .

Let $\Omega$ be a non-empty connected bounded open subset of $\mathbb{R}^{n}$ with Lipschitz boundary. Let $A=\left\{a_{i}\right\}_{i=1, \cdots, N}$ be a set of $N$ points of $\bar{\Omega}$ which contains a $P^{m-1}$-unisolvent subset. Let also $\left\{w_{i}\right\}_{i=1, \cdots, N}$ be a set of $N$ vectors of $\mathbb{R}^{n}$ that corresponds to the vector field dataset.

We aim at finding a function $\Phi: \bar{\Omega} \rightarrow \mathbb{R}$ smooth enough such that for $i=1, \cdots, N$,

$$
\nabla \Phi\left(a_{i}\right) \simeq w_{i} \in \mathbb{R}^{n} .
$$

Let us denote by $\rho$ the operator defined by:

$$
\rho:\left\{\begin{array}{l}
H^{m}\left(\Omega, \mathbb{R}^{n}\right) \rightarrow\left(\mathbb{R}^{n}\right)^{N} \\
v \mapsto \rho(v)=\left(v\left(a_{1}\right), \cdots, v\left(a_{N}\right)\right)^{T} .
\end{array}\right.
$$

Let us assume that $m>\frac{n}{2}$ so that the following Sobolev 's embeddings hold:

$$
\begin{aligned}
& H^{m}\left(\Omega, \mathbb{R}^{n}\right) \circlearrowleft \mathcal{C}^{0}\left(\bar{\Omega}, \mathbb{R}^{n}\right) \\
& H^{m+1}(\Omega, \mathbb{R}) \circlearrowleft \mathcal{C}^{1}(\bar{\Omega}, \mathbb{R})
\end{aligned}
$$

One sets $\forall \xi \in\left(\mathbb{R}^{n}\right)^{N}$ and $\forall \eta \in\left(\mathbb{R}^{n}\right)^{N}$,

$$
\langle\xi, \eta\rangle_{N}=\sum_{i=1}^{N}\left\langle\xi_{i}, \eta_{i}\right\rangle_{n}
$$

where $\langle\cdot, \cdot\rangle_{n}$ denotes the Euclidean scalar product in $\mathbb{R}^{n}$ and $\forall \xi \in\left(\mathbb{R}^{n}\right)^{N},\langle\xi\rangle_{N}=\langle\xi, \xi\rangle_{N}^{\frac{1}{2}}$. 


\section{Modelling}

\subsection{The fitting model}

We first introduce a regularized least-square problem defined on a space of potentials (realvalued functions) to fit the vector field dataset. For any $\varepsilon>0$, we introduce the functional $\mathcal{J}_{\varepsilon}$ defined as follows:

$$
\mathcal{J}_{\varepsilon}:\left\{\begin{array}{l}
H^{m+1}(\Omega, \mathbb{R}) \rightarrow \mathbb{R} \\
v \mapsto\langle\rho(\nabla v)-w\rangle_{N}^{2}+\varepsilon|v|_{m+1, \Omega, \mathbb{R}}^{2},
\end{array}\right.
$$

where $w=\left(w_{1}, \cdots, w_{N}\right)^{T} \in\left(\mathbb{R}^{n}\right)^{N}$ is the vector field dataset and $|\cdot|_{m+1, \Omega, \mathbb{R}}$, the semi-norm on $H^{m+1}(\Omega, \mathbb{R})$.

This kind of approach is related to the smoothing $D^{m}$-spline for surface approximation introduced by Arcangéli [4] (see also Duchon [12] for a general introduction or Gout [16], and López de Silanes and Arcangéli $[19,20]$ for convergence results). We consider the following fitting problem using potentials:

$$
\left\{\begin{array}{l}
\text { Search for } \sigma_{\varepsilon} \in H^{m+1}(\Omega, \mathbb{R}) \text { such that } \\
\forall v \in H^{m+1}(\Omega, \mathbb{R}), \mathcal{J}_{\varepsilon}\left(\sigma_{\varepsilon}\right) \leq \mathcal{J}_{\varepsilon}(v),
\end{array}\right.
$$

where $\mathcal{J}_{\varepsilon}$ is defined in (4). Obviously, the minimization problem (5) does not have a unique solution, the potential function $\sigma_{\varepsilon}$ being defined up to a constant.

Remark 2.1. An alternative model could be introduced to circumvent the lack of uniqueness of the potential function by including a few interpolation constraints in the modelling. Indeed, it would guarantee uniqueness of the solution expressed in terms of the potential function. In this case, the problem is phrased as a functional minimization problem on a convex subset $K$ (or a vector space if we retain only some positions for which the potential function is zero) of the Hilbert space $H^{m+1}(\Omega, \mathbb{R})$ as follows:

$$
\left\{\begin{array}{l}
\text { Search for } \sigma_{\epsilon} \in K \text { such that } \\
\forall v \in K, \mathcal{J}_{\epsilon}\left(\sigma_{\epsilon}\right) \leq \mathcal{J}_{\epsilon}(v)
\end{array}\right.
$$

with $K=\left\{v \in H^{m+1}(\Omega, \mathbb{R}), \forall i \in\{1, \cdots, M\}, v\left(b_{i}\right)=\beta_{i}\right\}$ and $\beta_{i}$ given at $b_{i}$ for all $i \in\{1, \cdots, M\}$. Existence and uniqueness of the solution are obtained by applying Stampacchia's theorem (after rewriting the functional to be minimized and using norm equivalence arguments resulting from Nečas theorem application). The introduction of Lagrange's multipliers allows to obtain the variational formulation of the problem on the whole space $H^{m+1}(\Omega, \mathbb{R})$ instead of a variational inequality. The main hindrance of this modelling comes from the fact that $\sigma_{\epsilon} \in H^{m+1}(\Omega, \mathbb{R})$. In practice $m=2$ in order to have $H^{3}(\Omega, \mathbb{R}) \circlearrowleft \mathcal{C}^{1}(\bar{\Omega}, \mathbb{R})$, yielding $\sigma_{\epsilon} \in H^{3}(\Omega, \mathbb{R})$. For instance, if we consider as generic finite element the $\mathcal{C}^{2}$ Bogner-Fox-Schmit element, there are 9 basis functions per node, leading to block matrices of size $81 \times 81$ to compute the stiffness matrix, which can turn out to be tricky from a numerical viewpoint. The idea of reformulating the problem in terms of the gradient vector field itself avoids this issue. 
So, in order to overcome the lack of uniqueness, we introduce the following problem expressed in terms of the gradients of the potentials: as $\varepsilon$ is a positive arbitrary parameter and as $|\cdot|_{m+1, \Omega, \mathbb{R}}$ and $|\nabla \cdot|_{m, \Omega, \mathbb{R}^{n}}$ are equivalent in $H^{m+1}(\Omega, \mathbb{R})$, we derive from problem $(5)$, the following problem of minimization:

$$
\left\{\begin{array}{l}
\text { Search for } \sigma_{\varepsilon} \in H^{m+1}(\Omega, \mathbb{R}) \text { such that } \forall v \in H^{m+1}(\Omega, \mathbb{R}), \\
\left\langle\rho\left(\nabla \sigma_{\varepsilon}\right)-w\right\rangle_{N}^{2}+\varepsilon\left|\nabla \sigma_{\varepsilon}\right|_{m, \Omega, \mathbb{R}^{n}}^{2} \leq\langle\rho(\nabla v)-w\rangle_{N}^{2}+\varepsilon|\nabla v|_{m, \Omega, \mathbb{R}^{n}}^{2} .
\end{array}\right.
$$

This formulation leads to the following problem associated to problem (6). To state this new problem, we first define the following functional denoted by $\mathcal{F}_{\varepsilon}$ :

$$
\mathcal{F}_{\varepsilon}:\left\{\begin{array}{l}
H^{m}\left(\Omega, \mathbb{R}^{n}\right) \rightarrow \mathbb{R} \\
f \mapsto\langle\rho(f)-w\rangle_{N}^{2}+\varepsilon|f|_{m, \Omega, \mathbb{R}^{n}}^{2},
\end{array}\right.
$$

and we consider the following problem stated by:

$$
\left\{\begin{array}{l}
\text { Search for } u_{\varepsilon} \in H^{m}\left(\Omega, \mathbb{R}^{n}\right) \text { such that } \\
\forall v \in H^{m}\left(\Omega, \mathbb{R}^{n}\right), \mathcal{F}_{\varepsilon}\left(u_{\varepsilon}\right) \leq \mathcal{F}_{\varepsilon}(v) .
\end{array}\right.
$$

This formulation is motivated by the following theorem (in the proof, we use the connectedness of $\Omega$ and the unisolvence property):

Theorem 2.2. If $\sigma_{\varepsilon}^{1}$ and $\sigma_{\varepsilon}^{2}$ are two distinct solutions of problem (6) then:

$$
\nabla \sigma_{\varepsilon}^{1}=\nabla \sigma_{\varepsilon}^{2}=u_{\varepsilon}
$$

where $u_{\varepsilon}$ is the unique solution of problem (8).

\subsection{Existence and uniqueness results}

We first give the following variational problem (which is equivalent to (8)):

$$
\left\{\begin{array}{l}
\text { Search for } u_{\varepsilon} \in H^{m}\left(\Omega, \mathbb{R}^{n}\right) \text { such that } \\
\forall v \in H^{m}\left(\Omega, \mathbb{R}^{n}\right),\left\langle\rho\left(u_{\varepsilon}\right), \rho(v)\right\rangle_{N}+\varepsilon\left(u_{\varepsilon}, v\right)_{m, \Omega, \mathbb{R}^{n}}=\langle w, \rho(v)\rangle_{N} .
\end{array}\right.
$$

We now give a lemma that will be of interest in the following.

Lemma 2.3. The mapping defined by:

$$
\|\cdot\|_{A, m, \Omega, \mathbb{R}^{n}}:\left\{\begin{array}{l}
H^{m}\left(\Omega, \mathbb{R}^{n}\right) \rightarrow \mathbb{R} \\
f \mapsto\|f\|_{A, m, \Omega, \mathbb{R}^{n}}=\left(\langle\rho(f)\rangle_{N}^{2}+|f|_{m, \Omega, \mathbb{R}^{n}}^{2}\right)^{\frac{1}{2}}=\left(\sum_{i=1}^{N}\left\langle f\left(a_{i}\right)\right\rangle_{n}^{2}+|f|_{m, \Omega, \mathbb{R}^{n}}^{2}\right)^{\frac{1}{2}}
\end{array}\right.
$$

is a Hilbert norm equivalent to the norm $\|f\|_{m, \Omega, \mathbb{R}^{n}}$ in $H^{m}\left(\Omega, \mathbb{R}^{n}\right)\left(H^{m}\left(\Omega, \mathbb{R}^{n}\right)\right.$ is endowed with the usual norm denoted by $\|\cdot\|_{m, \Omega, \mathbb{R}^{n}}$ and the associated semi-norm denoted by $|\cdot|_{m, \Omega, \mathbb{R}^{n}}$ ). 


\section{Proof .}

Let us take $f$ such that $\|f\|_{A, m, \Omega, \mathbb{R}^{n}}=0$. It implies that $|f|_{m, \Omega, \mathbb{R}^{n}}=0$ and taking into account the connectedness of $\Omega$, it yields $f \in P^{m-1}\left(\Omega, \mathbb{R}^{n}\right)$.

As the set $A$ contains a $P^{m-1}$-unisolvent subset, we deduce that $f \equiv 0$.

It is now clear that $\|\cdot\|_{A, m, \Omega, \mathbb{R}^{n}}$ is a norm on $H^{m}\left(\Omega, \mathbb{R}^{n}\right)$ associated with a scalar product.

We now prove the equivalence of the norm $\|\cdot\|_{A, m, \Omega, \mathbb{R}^{n}}$ with the norm $\|\cdot\|_{m, \Omega, \mathbb{R}^{n}}$. First, we have: $\forall f \in H^{m}\left(\Omega, \mathbb{R}^{n}\right), \forall a_{i} \in A, i=1, \cdots, N$,

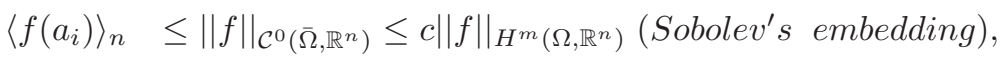

SO

$$
\|f\|_{A, m, \Omega, \mathbb{R}^{n}} \leq\left(1+c^{2} N\right)^{\frac{1}{2}}\|f\|_{m, \Omega, \mathbb{R}^{n}}
$$

Let us take $k=m$ and $p=2$ in Nečas' theorem (Chapter 2, section 7.1 from [23]) and let us take $\rho$ as functional $f_{i}$. Then, using the property of unisolvence of the set $A$, we obtain from Nečas' theorem that there exists a positive constant $c_{1}$ such that:

$$
\|f\|_{m, \Omega, \mathbb{R}^{n}} \leq \frac{1}{c_{1}}\left[|f|_{m, \Omega, \mathbb{R}^{n}}^{2}+\sum_{i=1}^{N}\left\langle f\left(a_{i}\right)\right\rangle_{n}^{2}\right]^{\frac{1}{2}}=\frac{1}{c_{1}}\|f\|_{A, m, \Omega, \mathbb{R}^{n}},
$$

which concludes the proof.

Using Lax-Milgram lemma and norm equivalence results, we can now establish the following theorem:

Theorem 2.4. Variational problem (10) admits a unique solution.

\subsection{Convergence result}

In the following, we consider classical convergence hypotheses (see [4] for more details).

Let $D$ be a subset of $\mathbb{R}^{+*}$ for which 0 is an accumulation point.

For any $d \in D$, let $A^{d}$ be a set of $N=N(d)$ distinct points from $\bar{\Omega}$ that contains a $P^{m-1}$-unisolvent subset. We assume that

$$
\sup _{x \in \Omega} \delta\left(x, A^{d}\right)=d
$$

where $\delta$ is the Euclidean distance in $\mathbb{R}^{n}$. Thus $d$ is the radius of the biggest sphere included in $\Omega$ that contains no point from $A^{d}$ (Hausdorff distance).

Also $d$ is bounded and

$$
\lim _{d \rightarrow 0} \sup _{x \in \Omega} \delta\left(x, A^{d}\right)=0 .
$$


For any $d \in D$, let us denote by $\rho^{d}$ the mapping defined by:

$$
\rho^{d}:\left\{\begin{array}{l}
H^{m}\left(\Omega, \mathbb{R}^{n}\right) \rightarrow\left(\mathbb{R}^{n}\right)^{N} \\
v \mapsto \rho^{d}(v)=\left((v(a))_{a \in A^{d}}\right)^{T} .
\end{array}\right.
$$

Introducing the norm $\|\cdot\|_{A^{d}, m, \Omega, \mathbb{R}^{n}}$ defined by:

$$
\|f\|_{A^{d}, m, \Omega, \mathbb{R}^{n}}=\left[\left\langle\rho^{d}(f)\right\rangle_{N}^{2}+|f|_{m, \Omega, \mathbb{R}^{n}}^{2}\right]^{\frac{1}{2}}
$$

as shown in Lemma 2.3 , the norm $\|\cdot\|_{A^{d}, m, \Omega, \mathbb{R}^{n}}$ is equivalent to the norm $\|\cdot\|_{m, \Omega, \mathbb{R}^{n}}$ in $H^{m}\left(\Omega, \mathbb{R}^{n}\right)$.

To prove the convergence result, we need the following lemma.

Lemma 2.5. Let $A_{0}=\left\{b_{01}, b_{02}, \cdots, b_{0 \aleph}\right\}$ be a fixed $P^{m-1}$-unisolvent subset of $\bar{\Omega}$ (in this case, $\left.\aleph=\operatorname{dim} P^{m-1}\right)$.

By hypothesis, $0 \in \bar{D}$ and (13) holds so:

$$
\forall j=1, \cdots, \aleph, \exists\left(a_{0 j}^{d}\right)_{d \in D},\left(\forall d \in D, a_{0 j}^{d} \in A^{d}\right) \quad \text { and }\left(b_{0 j}=\lim _{d \rightarrow 0} a_{0 j}^{d}\right) .
$$

For any $d \in D$, let $A_{0}^{d}$ be the set $\left\{a_{01}^{d}, \cdots, a_{0 \aleph}^{d}\right\}$ and let $\|\cdot\|_{A_{0}^{d}, m, \Omega, \mathbb{R}^{n}}$ be the mapping defined by: $\forall f \in H^{m}\left(\Omega, \mathbb{R}^{n}\right)$,

$$
\|f\|_{A_{0}^{d}, m, \Omega, \mathbb{R}^{n}}=\left[\sum_{j=1}^{\aleph}\left\langle f\left(a_{0 j}^{d}\right)\right\rangle_{n}^{2}+|f|_{m, \Omega, \mathbb{R}^{n}}^{2}\right]^{\frac{1}{2}} .
$$

Then, there exists $\eta>0$ such that for any $d \leq \eta$, the set $A_{0}^{d}$ is $P^{m-1}$-unisolvent and $\|\cdot\| \|_{A_{0}^{d}, m, \Omega, \mathbb{R}^{n}}$ is a norm on $H^{m}\left(\Omega, \mathbb{R}^{n}\right)$ uniformly equivalent over $\left.\left.D \cap\right] 0, \eta\right]$ to the norm $\|\cdot\|_{m, \Omega, \mathbb{R}^{n}}$.

\section{Proof}

The Sobolev's embedding gives that:

$$
\exists C_{1}>0, \forall d \in D, \forall f \in H^{m}\left(\Omega, \mathbb{R}^{n}\right),\|f\|_{A_{0}^{d}, m, \Omega, \mathbb{R}^{n}} \leq C_{1}\|f\|_{m, \Omega, \mathbb{R}^{n}} .
$$

The constant $C_{1}$ is independent of $d$ and depends on $\aleph$ which is the dimension of $P^{m-1}$.

Let us now find a constant $C_{2}$ independent of $d$ such that the inequality $\|f\|\left\|_{m, \Omega, \mathbb{R}^{n}} \leq C_{2}\right\| f \|_{A_{0}^{d}, m, \Omega, \mathbb{R}^{n}}$ holds.

For $f \in H^{m}\left(\Omega, \mathbb{R}^{n}\right)$,

$$
\begin{aligned}
\frac{1}{2} \sum_{j=1}^{\aleph}\left\langle f\left(b_{0 j}\right)\right\rangle_{n}^{2} & =\frac{1}{2} \sum_{j=1}^{\aleph}\left\langle f\left(b_{0 j}\right)-f\left(a_{0 j}^{d}\right)+f\left(a_{0 j}^{d}\right)\right\rangle_{n}^{2}, \\
\leq & \sum_{j=1}^{\aleph}\left\langle f\left(b_{0 j}\right)-f\left(a_{0 j}^{d}\right)\right\rangle_{n}^{2}+\sum_{j=1}^{\aleph}\left\langle f\left(a_{0 j}^{d}\right)\right\rangle_{n}^{2} .
\end{aligned}
$$


The open subset $\Omega$ having a Lipschitz-continuous boundary and $m$ being such that $m>\frac{n}{2}$, the space $H^{m}\left(\Omega, \mathbb{R}^{n}\right)$ satisfies the Sobolev's Hölder embedding theorem (continuous injection), namely:

$$
\exists \lambda \in] 0,1], H^{m}\left(\Omega, \mathbb{R}^{n}\right) \circlearrowleft \mathcal{C}^{0, \lambda}\left(\bar{\Omega}, \mathbb{R}^{n}\right) .
$$

Thus $f \in \mathcal{C}^{0, \lambda}\left(\bar{\Omega}, \mathbb{R}^{n}\right)$ and $\exists C>0, \forall j=1, \cdots, \aleph, \forall d \in D$,

$$
\left\langle f\left(b_{0 j}\right)-f\left(a_{0 j}^{d}\right)\right\rangle_{n}^{2} \leq\|f\|_{\mathcal{C}^{0, \lambda}\left(\bar{\Omega}, \mathbb{R}^{n}\right)}^{2}\left\langle b_{0 j}-a_{0 j}^{d}\right\rangle_{n}^{2 \lambda} \leq C^{2}\|f\|_{m, \Omega, \mathbb{R}^{n}}^{2}\left\langle b_{0 j}-a_{0 j}^{d}\right\rangle_{n}^{2 \lambda} .
$$

Besides, from (14) it comes: $\forall j=1, \cdots, \aleph$,

$$
\forall \beta_{j}>0, \exists \eta_{\beta_{j}}>0, \forall d \in D,\left(d \leq \eta_{\beta_{j}} \Rightarrow\left\langle a_{0 j}^{d}-b_{0 j}\right\rangle_{n} \leq \beta_{j}\right) .
$$

Then $\forall j=1, \cdots, \aleph$,

$$
\forall \beta_{j}>0, \exists \eta_{\beta_{j}}>0, \forall d \in D,\left(d \leq \eta_{\beta_{j}} \Rightarrow\left\langle f\left(b_{0 j}\right)-f\left(a_{0 j}^{d}\right)\right\rangle_{n}^{2} \leq C^{2}\|f\|_{m, \Omega, \mathbb{R}^{n}}^{2} \beta_{j}^{2 \lambda}\right) .
$$

Let $\beta>0$ and let us take $\beta_{j}=\beta, \forall j=1, \cdots, \aleph$ and $\eta=\min \left(\eta_{\beta_{1}}, \cdots, \eta_{\beta_{\aleph}}\right)$ then

$$
\forall d \in D, \quad\left(d \leq \eta \Rightarrow \sum_{j=1}^{\aleph}\left\langle f\left(b_{0 j}\right)-f\left(a_{0 j}^{d}\right)\right\rangle_{n}^{2} \leq C^{2} \beta^{2 \lambda} \aleph\|f\|_{m, \Omega, \mathbb{R}^{n}}^{2}\right) .
$$

This implies that:

$$
\begin{array}{r}
\forall \beta>0, \exists \eta>0, \forall d \in D, \forall f \in H^{m}\left(\Omega, \mathbb{R}^{n}\right), \\
\left(d \leq \eta \Rightarrow \frac{1}{2} \sum_{j=1}^{\aleph}\left\langle f\left(b_{0 j}\right)\right\rangle_{n}^{2}+|f|_{m, \Omega, \mathbb{R}^{n}}^{2}-C^{2} \beta^{2 \lambda} \aleph\|f\|_{m, \Omega, \mathbb{R}^{n}}^{2} \leq\|f\|_{A_{0}^{d}, m, \Omega, \mathbb{R}^{n}}^{2}\right)
\end{array}
$$

As previously shown, the mapping $f \in H^{m}\left(\Omega, \mathbb{R}^{n}\right) \mapsto\left[\frac{1}{2} \sum_{j=1}^{\aleph}\left\langle f\left(b_{0 j}\right)\right\rangle_{n}^{2}+|f|_{m, \Omega, \mathbb{R}^{n}}^{2}\right]^{\frac{1}{2}}$ is a norm on $H^{m}\left(\Omega, \mathbb{R}^{n}\right)$ equivalent to the norm $\|\cdot\|_{m, \Omega, \mathbb{R}^{n}}$ so:

$$
\begin{array}{r}
\forall \beta>0, \exists \eta>0, \forall d \in D, \forall f \in H^{m}\left(\Omega, \mathbb{R}^{n}\right), \\
\left(d \leq \eta \Rightarrow\left(C^{2}-C^{2} \beta^{2 \lambda} \aleph\right)\|f\|_{m, \Omega, \mathbb{R}^{n}}^{2} \leq\|f\|_{A_{0}^{d}, m, \Omega, \mathbb{R}^{n}}^{2}\right)
\end{array}
$$

By choosing $\beta$ adequately, the norm equivalence is obtained.

Theorem 2.6. Assume that there exists a function $\widehat{f} \in H^{m}\left(\Omega, \mathbb{R}^{n}\right)$ such that for any $d \in D$ : $\rho^{d}(\widehat{f})=w=w^{d}$ (we recall that $w=w^{d}$ is the given data set - a gradient vector set, that is, the input of our problem), and $\left.\varepsilon=\varepsilon(d) \in] 0, \varepsilon_{0}\right], \varepsilon_{0}>0$. For any $d \in D$, we denote by $u_{\varepsilon}^{d}$ the unique solution of problem (8). Then under the above assumptions we have:

$$
\lim _{d \rightarrow 0}\left\|u_{\varepsilon}^{d}-\widehat{f}\right\|_{m, \Omega, \mathbb{R}^{n}}=0
$$




\section{Proof 2.5.}

The proof is divided into four steps that we detail hereafter.

First step: We start by proving that the sequence $\left(u_{\varepsilon}^{d}\right)_{\substack{\left.d \in D \cap] 0, \eta] \\ \varepsilon \in] 0, \varepsilon_{0}\right]}}$ is bounded in $H^{m}\left(\Omega, \mathbb{R}^{n}\right)$ so that one can extract a subsequence $\left(u_{\varepsilon_{l}}^{d_{l}}\right)_{l \in \mathbb{N}}$ with $\lim _{l \rightarrow+\infty} d_{l}=0$ (since 0 is an accumulation point of $D$ ) and $\left.\left.\varepsilon_{l} \in\right] 0, \varepsilon_{0}\right], \forall l \in \mathbb{N}$ (-we assume that $\varepsilon=\varepsilon(d)$, so $\varepsilon_{l}=\varepsilon\left(d_{l}\right)$-) that weakly converges to an element of $H^{m}\left(\Omega, \mathbb{R}^{n}\right)$ denoted by $f^{*}$.

Second step: In the second step of the proof, we prove that $f^{*}=\widehat{f}$.

Third step: Then we aim at proving that the sequence $\left(u_{\varepsilon_{l}}^{d_{l}}\right)_{l \in \mathbb{N}}$ strongly converges to $\widehat{f}$ in $H^{m}\left(\Omega, \mathbb{R}^{n}\right)$. The Rellich-Kondrašov compact embedding theorem gives that:

$$
\forall r, r^{\prime} \in \mathbb{R}, r>r^{\prime}, H^{r}\left(\Omega, \mathbb{R}^{n}\right) \subset H^{r^{\prime}}\left(\Omega, \mathbb{R}^{n}\right) \text { (compact injection). }
$$

In our case, it means that the sequence $\left(u_{\varepsilon_{l}}^{d_{l}}\right)_{l \in \mathbb{N}}$ that weakly converges to $\widehat{f}$ in $H^{m}\left(\Omega, \mathbb{R}^{n}\right)$, strongly converges to $\widehat{f}$ in $H^{m-1}\left(\Omega, \mathbb{R}^{n}\right)$.

Therefore, as

$$
\left\|u_{\varepsilon_{l}}^{d_{l}}-\widehat{f}\right\|_{m, \Omega, \mathbb{R}^{n}}^{2}=\left\|u_{\varepsilon_{l}}^{d_{l}}-\widehat{f}\right\|_{m-1, \Omega, \mathbb{R}^{n}}^{2}+\left|u_{\varepsilon_{l}}^{d_{l}}-\widehat{f}\right|_{m, \Omega, \mathbb{R}^{n}}^{2},
$$

we just need to prove that

$$
\lim _{l \rightarrow \infty}\left|u_{\varepsilon_{l}}^{d_{l}}-\widehat{f}\right|_{m, \Omega, \mathbb{R}^{n}}=0
$$

One has:

$$
\begin{gathered}
\left|u_{\varepsilon_{l}}^{d_{l}}-\widehat{f}\right|_{m, \Omega, \mathbb{R}^{n}}^{2}=\left|u_{\varepsilon_{l}}^{d_{l}}\right|_{m, \Omega, \mathbb{R}^{n}}^{2}+|\widehat{f}|_{m, \Omega, \mathbb{R}^{n}}^{2}-2\left(u_{\varepsilon_{l}}^{d_{l}}, \widehat{f}\right)_{m, \Omega, \mathbb{R}^{n}} \\
\left|u_{\varepsilon_{l}}^{d_{l}}-\widehat{f}\right|_{m, \Omega, \mathbb{R}^{n}}^{2} \\
\leq 2|\widehat{f}|_{m, \Omega, \mathbb{R}^{n}}^{2}-2\left(u_{\varepsilon_{l}}^{d_{l}}, \widehat{f}\right)_{m, \Omega, \mathbb{R}^{n}}
\end{gathered}
$$

$\forall \alpha \in \mathbb{N}^{n},|\alpha|=m, \partial^{\alpha} u_{\varepsilon_{l}}^{d_{l}}$ weakly converges to $\partial^{\alpha} \widehat{f}$ in $L^{2}\left(\Omega, \mathbb{R}^{n}\right)$. So taking as test function $\widehat{f}$, it follows that $\lim _{l \rightarrow+\infty}\left(u_{\varepsilon_{l}}^{d_{l}}, \widehat{f}\right)_{m, \Omega, \mathbb{R}^{n}}=|\widehat{f}|_{m, \Omega, \mathbb{R}^{n}}^{2}$. Consequently,

$$
\lim _{l \rightarrow \infty}\left|u_{\varepsilon_{l}}^{d_{l}}-\widehat{f}\right|_{m, \Omega, \mathbb{R}^{n}}=0
$$

and finally

$$
\lim _{l \rightarrow \infty}\left\|u_{\varepsilon_{l}}^{d_{l}}-\widehat{f}\right\| \|_{m, \Omega, \mathbb{R}^{n}}=0
$$

Fourth step: Let us assume that $\left\|u_{\varepsilon}^{d}-f\right\|_{m, \Omega, \mathbb{R}^{n}}$ does not tend to 0 when $d$ tends to 0 .

It means that there exists a real number $\alpha>0$ and two sequences $\left(d_{k}\right)_{k \in \mathbb{N}}$ and $\left(\varepsilon_{k}\right)_{k \in \mathbb{N}}$ such that $d_{k} \rightarrow 0$ when $k \rightarrow+\infty$ and $\varepsilon_{k}=\varepsilon\left(d_{k}\right)$ and

$$
\forall k \in \mathbb{N},\left\|u_{\varepsilon_{k}}^{d_{k}}-\widehat{f}\right\|_{m, \Omega, \mathbb{R}^{n}}>\alpha .
$$


Following the same steps as previously done, there exists a subsequence of $\left(u_{\varepsilon_{k}}^{d_{k}}\right)_{k \in \mathbb{N}}$ that strongly converges to $\widehat{f}$ in $H^{m}\left(\Omega, \mathbb{R}^{n}\right)$. This last point is in contradiction with (20).

\section{Discretization}

\subsection{Introduction of the notations}

In this part, we give a discretization of the variational problem (10). We choose here a finite element space and we need the following notations.

- $\Delta$ is the dimension of $P^{m-1}$, space of $n$-variable polynomials with total degree less than $m-1$.

- Let $H$ be a bounded subset of $\mathbb{R}_{+}^{*}$ for which 0 is an accumulation point.

- Let $\tilde{\Omega}$ be a bounded polyhedral open subset of $\mathbb{R}^{n}$ containing $\Omega$.

- Let $m^{\prime}$ be an integer such that $m^{\prime}>m$. We recall that $m>\frac{n}{2}$.

- For any $h \in H$, let $\tilde{\mathcal{T}}_{h}$ be a triangulation of $\tilde{\Omega}$ by $n$-simplices or $n$-rectangles $K$ with diameter $h_{K} \leq h$ and let $\left(\tilde{V}_{h}\right)^{n}$ be a finite-element-type space constructed on $\tilde{\mathcal{T}}_{h}$, finite-dimensional subspace of $H^{m}\left(\tilde{\Omega}, \mathbb{R}^{n}\right)$. We assume that the family $\left(\tilde{T}_{h}\right)_{h \in H}$ is regular.

- $\forall h \in H$, let $\Omega_{h}$ be the open set defined as the interior of the union of elements $K \in \tilde{\mathcal{T}}_{h}$ such that $K \cap \tilde{\Omega} \neq \emptyset$. It can be seen that $\forall h \in H, \Omega \subset \Omega_{h} \subset \tilde{\Omega}$ and $\lim _{h \rightarrow 0} \operatorname{mes}\left(\Omega_{h} \backslash \Omega\right)=0$.

- For any $h \in H$, let $\left(V_{h}\right)^{n}$ be the space of restrictions to $\Omega_{h}$ of functions in $\left(\tilde{V}_{h}\right)^{n}$. It is a finite dimensional subspace of $H^{m}\left(\Omega_{h}, \mathbb{R}^{n}\right)$ with dimension $M=M(h)$.

We consider the variational formulation:

$$
\left\{\begin{array}{l}
\text { Find } u_{\varepsilon h}^{d} \in\left(V_{h}\right)^{n} \text { such that } \\
\forall v_{h} \in\left(V_{h}\right)^{n}, \\
\left\langle\rho^{d}\left(u_{\varepsilon h}^{d}\right), \rho^{d}\left(v_{h}\right)\right\rangle_{N=N(d)}+\varepsilon\left(u_{\varepsilon h}^{d}, v_{h}\right)_{m, \Omega_{h}, \mathbb{R}^{n}}=\left\langle w^{d}, \rho^{d}\left(v_{h}\right)\right\rangle_{N},
\end{array}\right.
$$

which is a discretisation of problem (10) and which admits a unique solution $u_{\varepsilon h}^{d}$. We aim to establish an error estimate for the solution $u_{\varepsilon h}^{d}$ of problem (10). We mainly use results from Duchon ([13]) and Apprato and Lopez de Silanes ([21]). We first recall two results from Duchon ([13]). For any $t \in \mathbb{R}^{n}$, and for $R>0$, we denote by $B(t, R)$ the closed ball centered at $t$ and with radius $R$. We have the following propositions (see Duchon [13] for the proofs):

Proposition 3.1. Let $\Omega$ be an open set of $\mathbb{R}^{n}$ with Lipschitz boundary. Then there exist constants $M>1, M_{1}>1$ and $\lambda_{0}>0$ such that, for any $\left.\left.\lambda \in\right] 0, \lambda_{0}\right]$, there exists $T_{\lambda} \subset \Omega$ such that:

$$
\left\{\begin{array}{cc}
i) & \forall t \in T_{\lambda}, B(t, \lambda) \subset \Omega \\
\text { ii) } & \Omega \subset \cup_{t \in T_{\lambda}} B(t, M \lambda) \\
\text { iii }) & \sum_{t \in T_{\lambda}} 1_{B(t, M \lambda)} \leq M_{1}
\end{array}\right.
$$


where $1_{E}$ is the characteristic function of the set $E$.

Proposition 3.2. There exist $R>0$ (depending on $n$ and $m$ ) and (for any $M \geq 1$ and any integer $l$ such that $l \leq m) C>0$ (depending on $M, m, n$ and $l$ ) satisfying: for any $d>0$ and any $t \in \mathbb{R}^{n}$, the ball $B(t, R d)$ contains $\Delta$ balls $\beta_{1}, \beta_{2}, \ldots, \beta_{\Delta}$ of radius $d$ such that:

$$
|u|_{l, B(t, M R d)} \leq C d^{m-l}|u|_{m, B(t, M R d)}
$$

for all $u \in H^{m}(B(t, M R d))$ that vanishes at at least one point of each ball $\beta_{i}, i \in\{1, \cdots, \Delta\}$.

The proof shows that the ball $B(t, R d)$ results from the mapping of a ball $B(\widehat{a}, R)$ by an affine transformation.

Let $\left\{\gamma_{1}^{0}, \gamma_{2}^{0}, \cdots, \gamma_{\Delta}^{0}\right\}$ be a $P^{m-1}$-unisolvent set. The set of $P^{m-1}$-unisolvent $\Delta$-tuples being an open set in $\left(\mathbb{R}^{n}\right)^{\Delta}$, there exists (see Duchon $\left.([13])\right) \delta>0$ such that $\left\{\gamma_{1}, \gamma_{2}, \ldots, \gamma_{\Delta}\right\}$ is still $P^{m-1}$ unisolvent if:

$$
\forall i=1, \cdots, \Delta,\left|\gamma_{i}-\gamma_{i}^{0}\right| \leq \delta
$$

By applying an homothety with ratio $\frac{1}{\delta}$ and setting $\widehat{\alpha}_{i}=\frac{1}{\delta} \gamma_{i}^{0}$, we obtain $\Delta$ balls $B\left(\widehat{\alpha}_{i}, 1\right)$ : the product $\prod_{i=1}^{\Delta} B\left(\widehat{\alpha}_{i}, 1\right)$ is a compact set of $\left(\mathbb{R}^{n}\right)^{\Delta}$ made up of $P^{m-1}$-unisolvent $\Delta$-tuples. The set $\bigcup_{i=1}^{\Delta} B\left(\widehat{\alpha}_{i}, 1\right)$ is bounded and thus included in a ball $B(\widehat{a}, R)$.

\subsection{Estimation of the approximation error $\widehat{f}-u_{\varepsilon h \mid \Omega}^{d}$}

Let $\widehat{f} \in H^{m^{\prime}}\left(\Omega, \mathbb{R}^{n}\right), m^{\prime}>m$ and let $u_{\varepsilon h \mid \Omega}^{d}\left(u_{\varepsilon h}^{d}\right.$ in the sequel) be the solution of problem (21) with $w^{d}=\rho^{d}(\widehat{f})$.

For any $t \in T_{R d} \in \Omega$, we define the mapping $F_{t}: x \mapsto t+d(x-\widehat{a})$, which is affine bijective and which maps the ball $B(\widehat{a}, M R)$ into the ball $B(t, M R d)$. Let $\left\{\widehat{a}_{1}, \widehat{a}_{2}, \cdots, \widehat{a}_{\Delta}\right\}$ be a subset of $B(\widehat{a}, R)$ such that $\widehat{a}_{i} \in B\left(\widehat{\alpha}_{i}, 1\right)$ and $F_{t}\left(\widehat{a}_{i}\right)=a_{i}^{d} \in A^{d}$, for $i=1, \cdots, \Delta$. This is made possible since the mapping $F_{t}$ maps the ball $B\left(\widehat{\alpha}_{i}, 1\right)$ into a ball of radius $d$ and since $d$ is defined by $(12)$. Note that the set $\left\{a_{1}^{d}, \cdots, a_{\Delta}^{d}\right\}$ is $P^{m-1}$-unisolvent. This property results from the closing remarks of subsection 3.1.

Let us set $v_{\varepsilon}=\widehat{f}-u_{\varepsilon h}^{d}$ and $\widetilde{v_{\varepsilon}}=P v_{\varepsilon}$, where $P$ is the $m$-extension operator of $H^{m}\left(\Omega, \mathbb{R}^{n}\right)$ into $H^{m}\left(\mathbb{R}^{n}, \mathbb{R}^{n}\right)$.

For any $t \in T_{R d}$, let $\Pi_{t}$ be the $P^{m-1}$-Lagrange interpolation operator such that for $\widetilde{v_{\varepsilon}} \in H^{m}\left(B(t, M R d), \mathbb{R}^{n}\right)$,

$$
\Pi_{t} \widetilde{v_{\varepsilon}} \in P^{m-1} \text { and } \Pi_{t} \widetilde{v_{\varepsilon}}\left(a_{i}^{d}\right)=\widetilde{v_{\varepsilon}}\left(a_{i}^{d}\right), i=1, \cdots, \Delta .
$$

We have the following propositions (see Lopez de Silanes and Apprato ([21]) for the adaptation of the proofs to our problem): 
Proposition 3.3. There exist positive constants $R, \lambda_{0}, \theta$ and $C$ such that for all $d \leq \min \left(\frac{\lambda_{0}}{R}, \theta\right)$, for all $\varepsilon$ and for all $h$, one has:

$$
\forall l=0, \cdots, m, \sum_{t \in T_{R d}}\left|\Pi_{t} \widetilde{v}_{\varepsilon}\right|_{l, B(t, M R d), \mathbb{R}^{n}}^{2} \leq C^{2} d^{n-2 l} \varepsilon
$$

Proposition 3.4. There exist positive constants $R, \lambda_{0}, \eta$ and $C$ such that for all $d \leq \min \left(\frac{\lambda_{0}}{R}, \eta\right)$, for all $\varepsilon$ and for all $h$, one has:

$$
\forall l=0, \cdots, m, \sum_{t \in T_{R d}}\left|\widetilde{v}_{\varepsilon}-\Pi_{t} \widetilde{v}_{\varepsilon}\right|_{l, B(t, M R d), \mathbb{R}^{n}}^{2} \leq C^{2}\left|v_{\varepsilon}\right|_{m, \Omega, \mathbb{R}^{n}}^{2} d^{2(m-l)} .
$$

Theorem 3.5. Let $\widehat{f} \in H^{m^{\prime}}\left(\Omega, \mathbb{R}^{n}\right), m^{\prime}>m$ and let $u_{\varepsilon h}^{d}$ be the solution of (21) with $w^{d}=\rho^{d}(\widehat{f})$. Then there exist positive constants $R, \lambda_{0}, \theta, \eta$ and $C$ such that for all $d \leq \min \left(\frac{\lambda_{0}}{R}, \theta, \eta\right)$, for all $\varepsilon$ and for all $h$, one has:

$$
\forall l=0, \cdots, m,\left|\widehat{f}-u_{\varepsilon h}^{d}\right|_{l, \Omega, \mathbb{R}^{n}} \leq C\left[\left|\widehat{f}-u_{\varepsilon h}^{d}\right|_{m, \Omega, \mathbb{R}^{n}} d^{m-l}+d^{\frac{n}{2}-l} \varepsilon^{\frac{1}{2}}\right] .
$$

As a consequence, when $u_{\varepsilon h}^{d}$ converges to $\widehat{f}$ in $H^{m}\left(\Omega, \mathbb{R}^{n}\right)$ (which is the case under some additional assumptions), one has:

$$
\begin{array}{r}
\forall l=0, \cdots, m-1,\left|\widehat{f}-u_{\varepsilon h}^{d}\right|_{l, \Omega, \mathbb{R}^{n}}=o\left(d^{m-l}\right)+O\left(d^{\frac{n}{2}-l} \varepsilon^{\frac{1}{2}}\right), \\
\left|\widehat{f}-u_{\varepsilon h}^{d}\right|_{l, \Omega, \mathbb{R}^{n}}=o(1)+O\left(d^{\frac{n}{2}-l} \varepsilon^{\frac{1}{2}}\right) \text { since }\left|\widehat{f}-u_{\varepsilon h}^{d}\right|_{l, \Omega, \mathbb{R}^{n}} \leq C\left[\| \widehat{f}-\left.u_{\varepsilon h}^{d}\right|_{m, \Omega, \mathbb{R}^{n}}+d^{\frac{n}{2}-l} \varepsilon^{\frac{1}{2}}\right] .
\end{array}
$$

This error estimate is only significant when the term $d^{n / 2-l} \varepsilon^{1 / 2}$ tends to zero.

Let us note that Theorem 3.5 only gives a convergence result based on the density $(d)$ of the given data samples. Of course, it is also possible to link the finite element parameter $h$ with a convergence result, and therefore to link both $d$ and $h$ in a theoretical convergence result.

In effect, from Theorem 3.5, it is possible to obtain error estimates (depending on $h$ ) on $\left|\widehat{f}-u_{\epsilon h}^{d}\right|_{l, \Omega, \mathbb{R}^{n}}: l=0, \ldots, m$. To do that, we need the following hypotheses:

$$
\exists C>0, \exists \Theta>0, \forall d \in D,\left(d \leq \Theta \Rightarrow N(d) \leq \frac{C}{d^{n}}\right) .
$$

This hypothesis implies an asymptotic distribution of the data on $\bar{\Omega}$ (see Arcangeli et al. [4]). The generic finite elements $\left(K, P_{K}, \Sigma_{K}\right)$ of $\left(\widetilde{V}_{h}\right)_{h \in H}$ must satisfy

$$
P_{K} \supset P^{m^{\prime}-1}(\bar{\Omega})
$$

and 


$$
\left\{\begin{array}{l}
\forall v \in H^{m^{\prime}}\left(\widetilde{\Omega}, \mathbb{R}^{n}\right), \exists\left(v_{h}\right)_{h \in H} \subset\left(\widetilde{V}_{h}\right)^{n}, \forall h \in H, \forall l=0, \ldots, m, \\
\left|v-v_{h}\right|_{l, \Omega, \mathbb{R}^{n}} \leq C h^{m^{\prime}-l}|v|_{m^{\prime}, \widetilde{\Omega}, \mathbb{R}^{n}} .
\end{array}\right.
$$

Let us note that inequation (24) is needed to establish the convergence of the approximation but it does not require the usual regularity hypothesis of the finite element method: $H^{m^{\prime}}\left(\widetilde{\Omega}, \mathbb{R}^{n}\right) \hookrightarrow$ $C^{s}\left(\overline{\widetilde{\Omega}}, \mathbb{R}^{n}\right)$. Of course, hypothesis (24) requires that the triangulation is regular (see Ciarlet [9]). These hypotheses (23-24) are satisfied in the usual finite element framework (see Clément [10], Strang [24]).

We also consider $\widetilde{\widehat{f}} \in H^{m^{\prime}}\left(\widetilde{\Omega}, \mathbb{R}^{n}\right)$ a $m^{\prime}-$ extension of $\widehat{f}$ on $\widetilde{\Omega}$ (existing since $\Omega$ has a Lipschitz boundary):

$$
\widetilde{\widehat{f}}_{\mid \Omega}=\widehat{f} \text { in } H^{m^{\prime}}\left(\Omega, \mathbb{R}^{n}\right),
$$

with $H^{m^{\prime}}\left(\Omega, \mathbb{R}^{n}\right) \circlearrowleft C^{0}\left(\bar{\Omega}, \mathbb{R}^{n}\right)$.

Then we have the following theorem:

Theorem 3.6. We keep the hypotheses of Theorem 3.5, and we suppose satisfied the hypotheses (22-24). Then, there exists $C>0$ such that: for any $d \leq \min \left(\frac{\lambda_{0}}{R}, \Theta, \eta\right)$, for any $\varepsilon>0$, and for any $h \in H$, we have:

$$
\left|\widehat{f}-u_{\epsilon h}^{d}\right|_{l, \Omega, \mathbb{R}^{n}} \leq C\left[\frac{h^{m^{\prime}-m} d^{m-l-n / 2}}{\sqrt{\varepsilon}}+\left(h^{m^{\prime}-m}+\sqrt{2}\right)|\widetilde{\hat{f}}|_{m^{\prime}, \widetilde{\Omega}, \mathbb{R}^{n}} d^{m-l}+d^{-l+n / 2} \varepsilon^{1 / 2}\right],
$$

where $\widetilde{\widehat{f}}$ is introduced in (25).

Remark 3.7. Let us note that we could use the relation between $d$ and $h$ to find a non-uniform triangulation adapted to the given data. But in this paper, we assume that we have no a priori knowledge of the dataset distribution. Therefore, a uniform triangulation seems to be the best tradeoff (see [4]).

\section{Experimental results.}

We conclude the paper by presenting several numerical experiments on both synthetic datasets and real data. The experiments have been performed on a 2.21-GHz Athlon with $1.00 \mathrm{~GB}$ of RAM.

In this part, we give a discretization of the variational problem (10). In the following, $\Omega$ is a rectangle, and we limit ourselves to the case $n=2$ and $m=2$ so that the Sobolev's embedding $H^{2}\left(\Omega, \mathbb{R}^{2}\right) \circlearrowleft \mathcal{C}^{0}\left(\bar{\Omega}, \mathbb{R}^{2}\right)$ holds.

We keep the notations introduced in Sections 2 and 3. We solve (10) on a finite element space $\left(V_{h}\right)^{2} \subset H^{2}\left(\Omega, \mathbb{R}^{2}\right)$ whose generic finite element is the $\mathcal{C}^{1}$ rectangular Bogner-Fox-Schmit (BFS) element.

If $\left(K, P_{K}, \Sigma_{K}\right)$ is the generic $\mathcal{C}^{1}$ BFS element, the domain $\Omega$ is triangulated by means of the rectangles $K$.

It can be proved that problem (10) uncouples with respect to each component in $\mathbb{R}^{2}$. 
Let $\left(v^{q}\right)_{q=1,2}$ be the components of $v \in H^{2}\left(\Omega, \mathbb{R}^{2}\right)$ and $\left(w_{i}^{q}\right)_{q=1,2}$ be the components of $w_{i} \in \mathbb{R}^{2}$, $\forall i \in\{1, \cdots, N\}$. Problem (10) can therefore be stated by:

$$
\left\{\begin{array}{l}
\text { Search for } u_{\epsilon}=\left(u_{\epsilon}^{q}\right)_{q=1,2} \in H^{2}\left(\Omega, \mathbb{R}^{2}\right) \text { such that } \\
\forall v=\left(v^{q}\right)_{q=1,2} \in H^{2}\left(\Omega, \mathbb{R}^{2}\right), \\
\forall q=1,2 \\
\sum_{i=1}^{N} u_{\epsilon}^{q}\left(a_{i}\right) v^{q}\left(a_{i}\right)+\epsilon\left(u_{\epsilon}^{q}, v^{q}\right)_{2, \Omega, \mathbb{R}}=\sum_{i=1}^{N} w_{i}^{q} v^{q}\left(a_{i}\right) .
\end{array}\right.
$$

We solve (26) in $V_{h}$ for $q=1,2$. Let $M_{h}$ be the dimension of $V_{h}$ and $\left\{P_{j}^{h}\right\}_{j=1, \cdots, M_{h}}$ be the basis functions. If we denote by $u_{\epsilon}^{h, q}$ the solution of problem (26) approximated in $\left(V_{h}\right)^{2}$, we can decompose $u_{\epsilon}^{h, q}$ into the basis $\left\{P_{j}^{h}\right\}_{j=1, \cdots, M_{h}}$ :

$$
\left\{\begin{array}{l}
\forall q=1,2 \\
\exists\left(\alpha_{j}^{q}\right)_{j=1, \cdots, M_{h}} \in \mathbb{R} \\
u_{\epsilon}^{h, q}=\sum_{j=1}^{M_{h}} \alpha_{j}^{q} P_{j}^{h}
\end{array}\right.
$$

For $q=1,2$, taking successively $v^{q}=P_{l}^{h}, l=1, \cdots, M_{h}$ in (26), the studied problem is rephrased by:

$$
\left\{\begin{array}{l}
\text { Search for } \alpha^{q} \in \mathbb{R}^{M_{h}} \text { such that, } \\
\forall l=1, \cdots, M_{h}, \\
\sum_{i=1}^{N} \sum_{j=1}^{M_{h}} \alpha_{j}^{q} P_{j}^{h}\left(a_{i}\right) P_{l}^{h}\left(a_{i}\right)+\epsilon \sum_{j=1}^{M_{h}} \alpha_{j}^{q}\left(P_{j}^{h}, P_{l}^{h}\right)_{2, \Omega, \mathbb{R}}=\sum_{i=1}^{N} w_{i}^{q} P_{l}^{h}\left(a_{i}\right) .
\end{array}\right.
$$

Denoting by $A^{h}$ and $R^{h}$ the matrices defined by:

$$
\begin{aligned}
& A^{h}=\left(P_{j}^{h}\left(a_{i}\right)\right)_{1 \leq i \leq N, 1 \leq j \leq M_{h}} \in \mathcal{M}_{N \times M_{h}}(\mathbb{R}), \\
& R^{h}=\left(\left(P_{j}^{h}, P_{i}^{h}\right)_{2, \Omega, \mathbb{R}}\right)_{1 \leq i \leq M_{h}, 1 \leq j \leq M_{h}} \in \mathcal{M}_{M_{h} \times M_{h}}(\mathbb{R}),
\end{aligned}
$$

we are led to solve two uncoupled sparse linear subsystems that can be written in the form:

$$
\left(\left(A^{h}\right)^{T} A^{h}+\epsilon R^{h}\right) \alpha^{q}=\left(A^{h}\right)^{T} w^{q}, \forall q=1,2,
$$

with $w^{q} \in \mathbb{R}^{N}, \forall q=1,2$. It can be easily demonstrated that these systems admit a unique solution. The matrix that intervenes in both systems is symmetric, positive definite, and independent of $q$. Only the second member differs in both systems. 
In each test, the parameter $\epsilon$ has been set to $10^{-6}$.

Also, in order to provide a quantitative measure of the degree of approximation, we have computed for each example the relative error $\chi$ defined as follows:

$$
\chi=\left(\frac{\sum_{i=1}^{N}\left\langle u_{\epsilon}^{h}\left(a_{i}\right)-w_{i}\right\rangle_{2}^{2}}{\sum_{i=1}^{N}\left\langle w_{i}\right\rangle_{2}^{2}}\right)^{\frac{1}{2}} .
$$

We now propose several examples to illustrate the efficiency of the proposed method.

\subsection{Experimental tests on synthetic datasets}

In the first experiment, we are concerned with the function $f$ defined on $\Omega=(0,1) \times(0,1)$ by $f(x, y)=\exp \left(-(3 x-1)^{2}-(3 y-1)^{2}\right)$. To apply our method, we have generated a fixed set of points $A=\left\{a_{1}, \cdots, a_{N}\right\}$ regularly distributed on $\bar{\Omega}$ and have set: $\forall i=1, \cdots, N, w_{i}=\nabla f\left(a_{i}\right)$. The set $A$ consists of 1681 points. The triangulation of the domain $\Omega$ is made up of $8 \times 8$ equal squares and so $M_{h}=4 \times 9^{2}=324$. The fixed set of points $A$ is depicted on Fig. 1, left column, and the given vector field $\left\{w_{i}\right\}_{i=1, \cdots, N}$ on Fig. 1, right column. The reference frame is centred on the bottom left corner. The $x$-direction corresponds to the column direction while the $y$-direction corresponds to the row direction and points upwards. Also, this figure is complemented by the
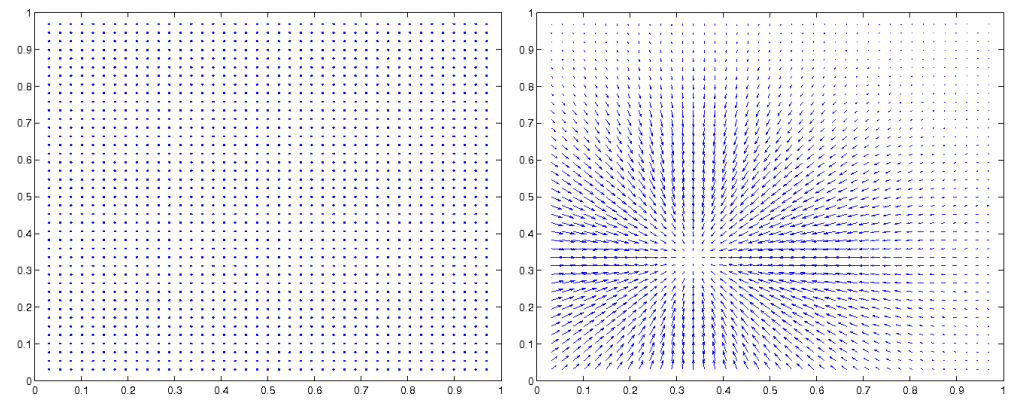

Figure 1: On the left, the regularly distributed fixed set of points $A$. On the right, the given vector field. Note that the Matlab function used to display the field scales the arrows to fit within the grid.

plots of each component of the vector field, Fig. 2. The finite element space $V_{h}$ is constructed on the triangulation from the Bogner-Fox-Schmit rectangle of class $\mathcal{C}^{1}$ and the corresponding approximant $u_{\epsilon}^{h}$ related to $A$ is computed. The results are presented in Fig. $3-4$. We have used a mesh refinement for the visualization: each square has been subdivided into $10 \times 10$ equal squares. As shown, the approximant proves to be satisfactory: the relative error is equal to 0.0003946990 . To measure the accuracy of the method, we have then successively removed (randomly) 25\%, 50\%, $75 \%$ and $85 \%$ of the data and have computed the relative error only on the missing data. The obtained results are displayed in Table 1. 

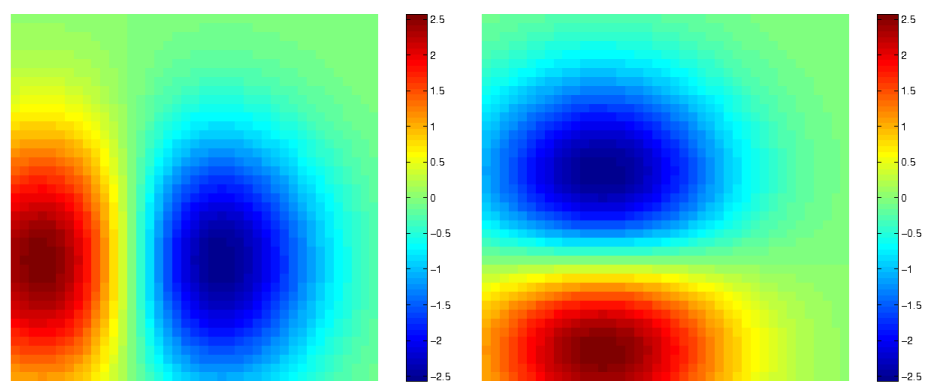

Figure 2: On the left, plot of the first component of the vector field $\left\{w_{i}\right\}_{i=1, \cdots, N}$. On the right, plot of the second component.
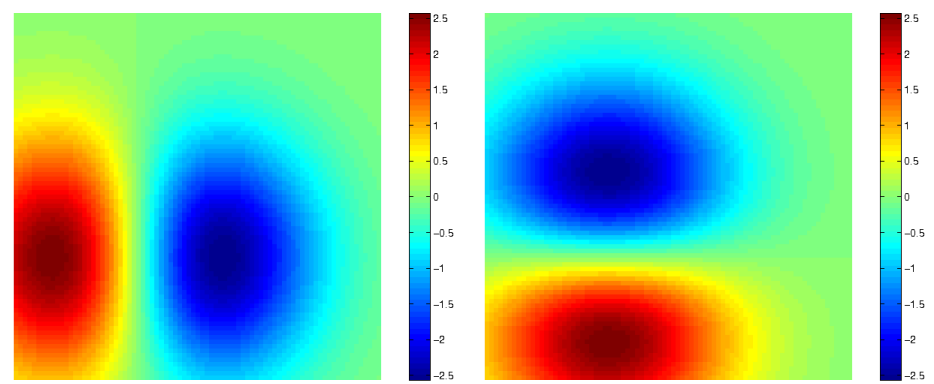

Figure 3: On the left, plot of the first component of the obtained vector field. On the right, plot of the second component.

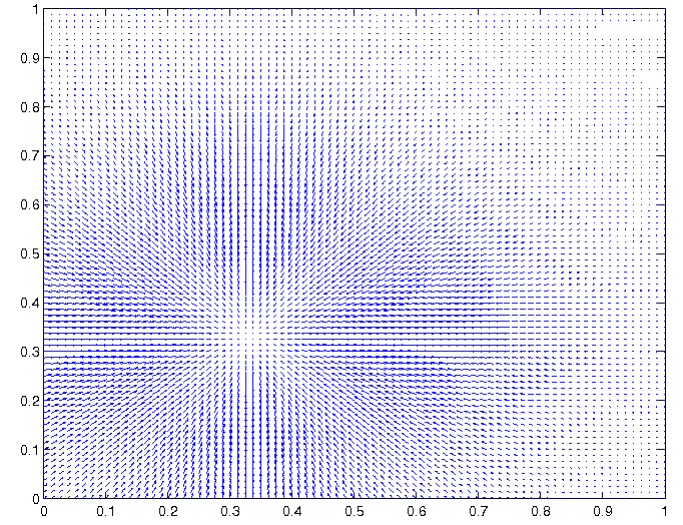

Figure 4: Plot of the obtained vector field. 


\begin{tabular}{|c|c|}
\hline \multicolumn{2}{|c|}{ Test 1 on synthetic dataset } \\
\hline Percentage of remaining data & Relative error \\
\hline 75 & 0.000471 \\
\hline 50 & 0.000590 \\
\hline 25 & 0.002217 \\
\hline 15 & 0.004729 \\
\hline
\end{tabular}

Table 1: Relative error when some data are randomly removed. (Fixed triangulation).

We now propose to illustrate numerically the theoretical convergence result provided in Theorem 3.5. To do that, we consider $\varepsilon(d)=d^{5}$. The theoretical error related to $\left|\widehat{f}-u_{\epsilon h}^{d}\right|_{0, \Omega, \mathbb{R}^{2}}$ should be of order $O\left(d^{7 / 2}\right)$.

\begin{tabular}{|c|c|c|c|}
\hline$d$ & $\varepsilon$ & Error & \% of data \\
\hline 0.0442 & $1.6810-7$ & $9.828110-4$ & 100 \\
\hline 0.0630 & $9.91310-7$ & $8.728410-4$ & 75 \\
\hline 0.0718 & $1.911510-6$ & 0.0014 & 50 \\
\hline 0.0954 & $7.88710-6$ & 0.0099 & 25 \\
\hline 0.1063 & $1.354810-5$ & 0.0165 & 15 \\
\hline 0.1978 & $3.027610-4$ & 0.1496 & 5 \\
\hline 0.3815 & 0.0081 & 0.6248 & 1 \\
\hline
\end{tabular}

The linear regression associated with the data $X=\log (d)$ and $Y=\log ($ error $)$ gives as slope: 3.42. This coefficient is very close to the coefficient given by Theorem 3.6 : 3.5. 
In the second experiment, we focus on Franke's function defined on $\Omega=(0,1) \times(0,1)$ by:

$$
\begin{aligned}
f(x, y) & =0.75 \exp \left(-0.25(9 x-2)^{2}-0.25(9 y-2)^{2}\right)+0.75 \exp \left(-(9 x+1)^{2} / 49-(9 y+1)^{2} / 10\right) \\
& +0.5 \exp \left(-0.25(9 x-7)^{2}-0.25(9 y-3)^{2}\right)-0.2 \exp \left(-(9 x-4)^{2}-(9 y-7)^{2}\right) .
\end{aligned}
$$

As previously done, we have generated a fixed set of points $A=\left\{a_{1}, \cdots, a_{N}\right\}$ regularly distributed on $\bar{\Omega}$ and have set $\forall i=1, \cdots, N, w_{i}=\nabla f\left(a_{i}\right)$. The set $A$ consists of 1681 points. The triangulation of the domain $\Omega$ is made up of $8 \times 8$ equal squares and so $M_{h}=4 \times 9^{2}=324$. The fixed set of points $A$ is depicted on Fig. 5, left column, and the given vector field $\left\{w_{i}\right\}_{i=1, \cdots, N}$ on Fig. 5, right column. The reference frame is centred on the bottom left corner. The $x$-direction corresponds to the column direction while the $y$-direction corresponds to the row direction and points upwards.
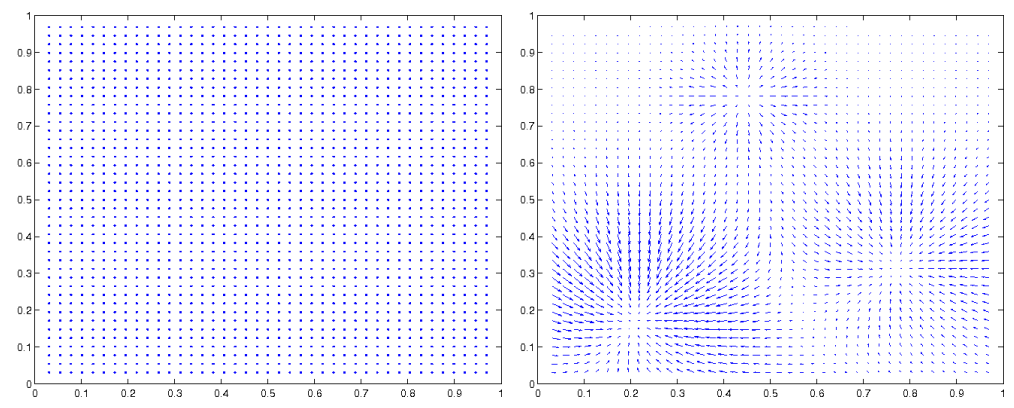

Figure 5: On the left, the regularly distributed fixed set of points $A$. On the right, the given vector field. Note that the Matlab function used to display the field scales the arrows to fit within the grid.

Also, this figure is complemented by the plots of each component of the vector field, Fig. 6 . The finite element space $V_{h}$ is constructed on the triangulation from the Bogner-Fox-Schmit rectangle of class $\mathcal{C}^{1}$ and the corresponding approximant $u_{\epsilon}^{h}$ related to $A$ is computed.
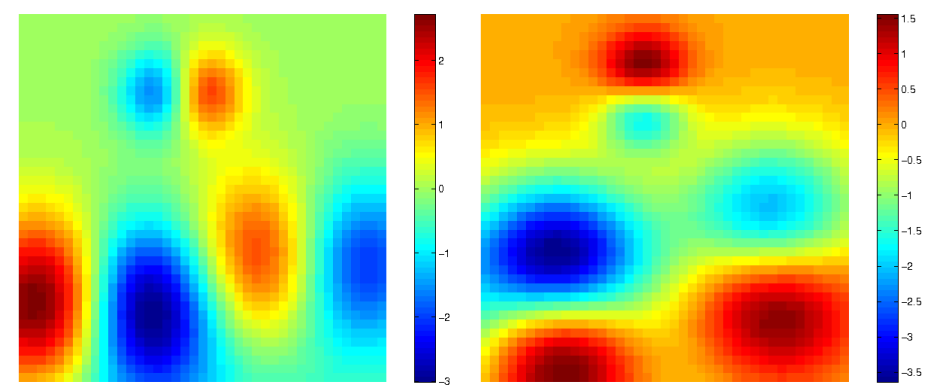

Figure 6: On the left, plot of the first component of the vector field $\left\{w_{i}\right\}_{i=1, \cdots, N}$. On the right, plot of the second component.

The results are presented in Fig. 7-8. We have used a mesh refinement for the visualization: each square has been subdivided into $20 \times 20$ equal squares. The relative error is equal to 
0.0035175393. To measure the accuracy of the method, we have then successively removed (randomly) $25 \%, 50 \%, 75 \%$ and $85 \%$ of the data and have computed the relative error only on the missing data. The obtained results are displayed in Table 2.

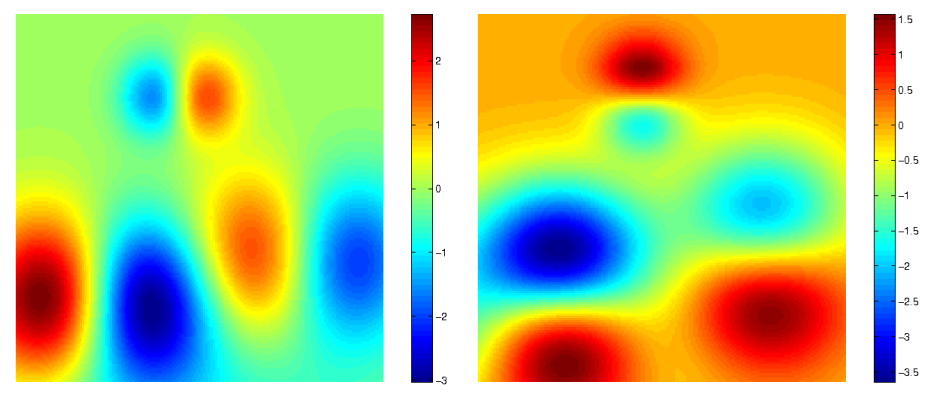

Figure 7: On the left, plot of the first component of the obtained vector field. On the right, plot of the second component.

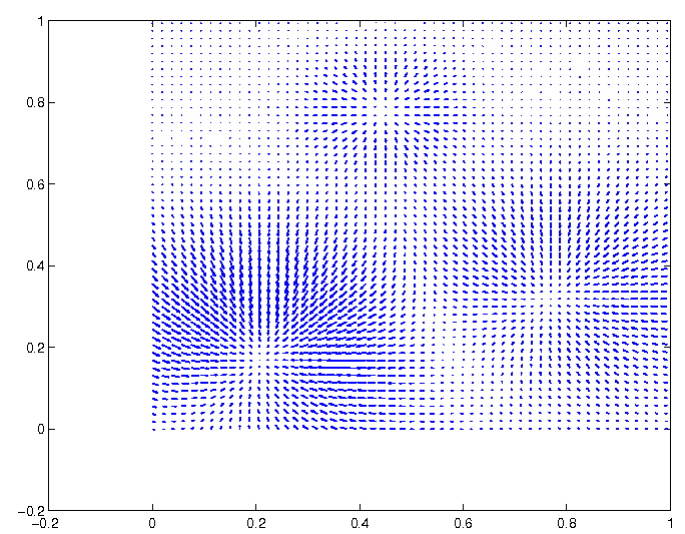

Figure 8: Plot of the obtained vector field every 3 rows and columns.

\begin{tabular}{|c|c|}
\hline \multicolumn{2}{|c|}{ Test 2 on synthetic dataset } \\
\hline Percentage of remaining data & Relative error \\
\hline 75 & 0.004265 \\
\hline 50 & 0.004455 \\
\hline 25 & 0.008983 \\
\hline 15 & 0.028743 \\
\hline
\end{tabular}

Table 2: Relative error when some data are randomly removed. (Fixed triangulation). 


\subsection{Datasets of wind velocity fields}

We focus on data of wind velocity fields that have been acquired in the Mozambique Channel. The Mozambique Channel is a portion of the Indian Ocean located between the island of Madagascar and Southeast Africa (see Fig. 9). These data have been measured by the satellite ERS2. They have been smoothed and monthly averaged by the CERSAT laboratory (IFREMER-France). No data have been recorded on the land so we have set them to 0 . In the first experiment, the data were acquired in January. In January, the monsoon is present and induces a north wind in the north part of the channel. The south-east wind present in the south part of the channel corresponds to trade winds (see [22] for more details). The data are regularly distributed on the domain $\Omega$. The set $A$ consists of 528 points. The triangulation of the domain $\Omega$ is made up of $15 \times 15$ rectangles and so $M_{h}=4 \times 16^{2}=1024$. The given set of vectors $\left\{w_{i}\right\}_{i=1, \cdots, N}$ is depicted in Fig. 10.

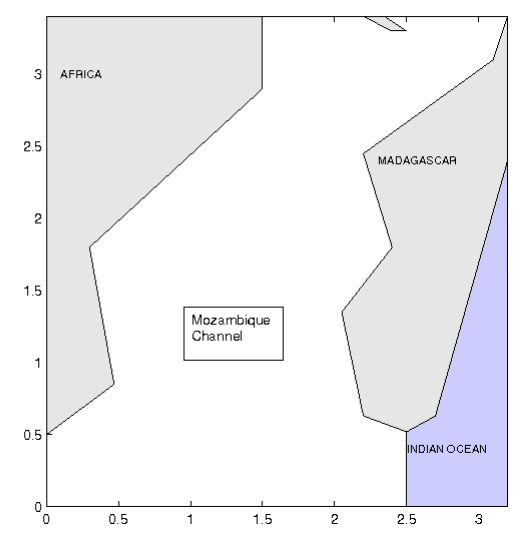

Figure 9: Depiction of the Mozambique Channel.

Also, this figure is complemented by the plots of each component of the vector field, Fig. 11. The finite element space $V_{h}$ is constructed on the triangulation from the Bogner-Fox-Schmit rectangle of class $\mathcal{C}^{1}$ and the corresponding approximant $u_{\epsilon}^{h}$ is computed. The results are presented in Fig. 12-13. We have used a mesh refinement for the visualization: each rectangle has been subdivided into $20 \times 20$ rectangles. The relative error is equal to 0.0001650361 which is considered as a very good result in this context. We removed $25 \%$ of the data (randomly) and computed the relative error at all data locations. It reached 0.076156 . For higher percentages, as the number of data is rather small, we do not get as good results as for the synthetic data: the relative error is in $10^{-1}$.

The second example concentrates upon the same kind of data. The processed data were acquired in August. In August, the monsoon does not exist and the wind field is dominated by trade winds. The set $A$ consists of 528 points. The triangulation of the domain $\Omega$ is made up of $15 \times 15$ rectangles and so $M_{h}=4 \times 16^{2}=1024$. The given set of vectors $\left\{w_{i}\right\}_{i=1, \ldots, N}$ is depicted in Fig. 14. Also, this figure is complemented by the plots of each component of the vector field, Fig. 15. The finite element space $V_{h}$ is constructed on the triangulation from the Bogner-Fox-Schmit rectangle of class 


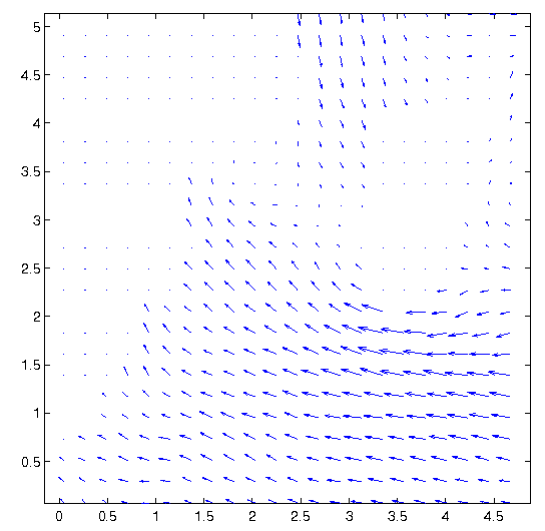

Figure 10: Plot of the given vector field $\left\{w_{i}\right\}_{i=1, \cdots, N}$.
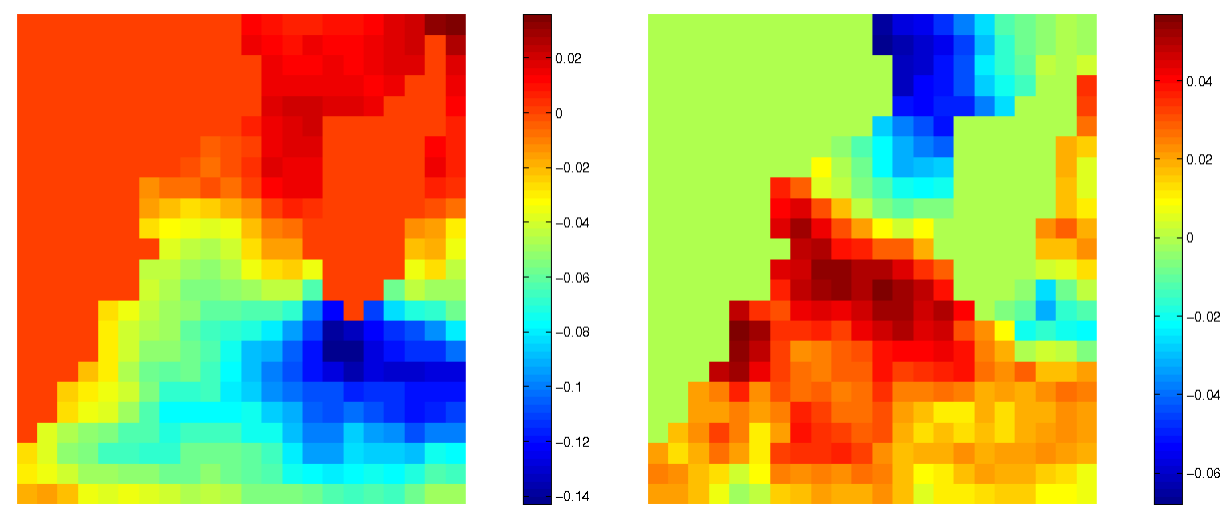

Figure 11: On the left, plot of the first component of the vector field $\left\{w_{i}\right\}_{i=1, \cdots, N}$. On the right, plot of the second component. 

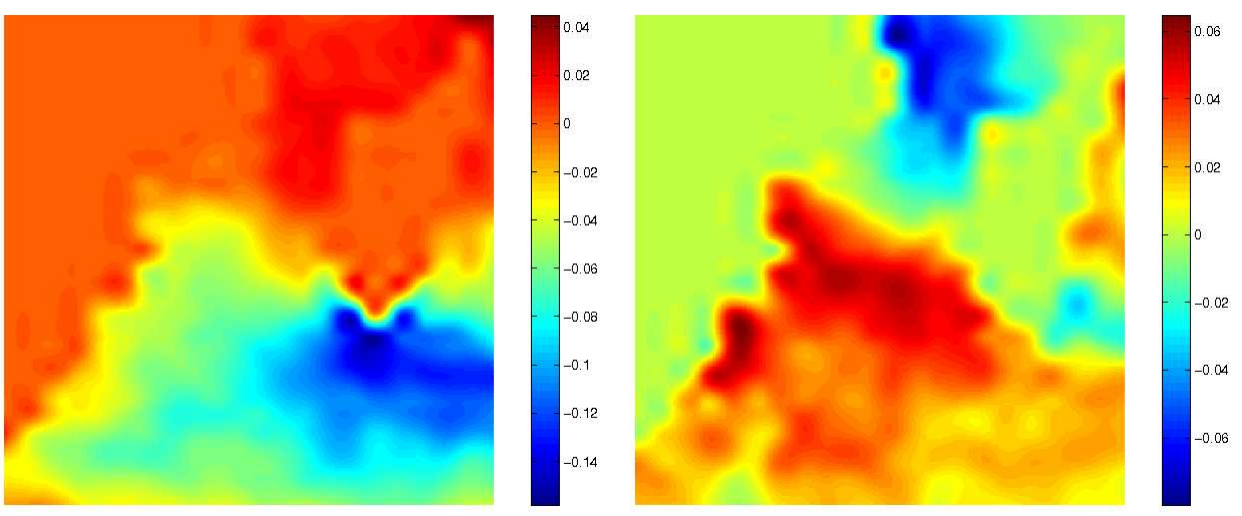

Figure 12: On the left, plot of the first component of the obtained vector field. On the right, plot of the second component.

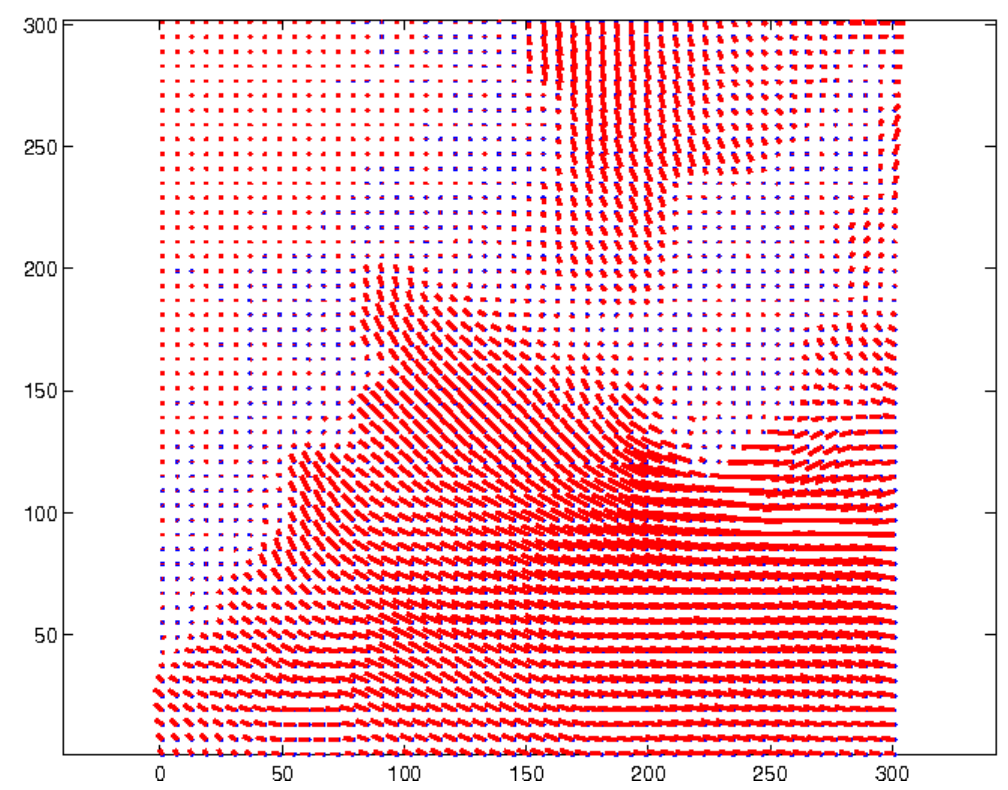

Figure 13: Plot of the obtained vector field (every six rows and columns). The blue diamond indicates the point to which the vector is attached. 


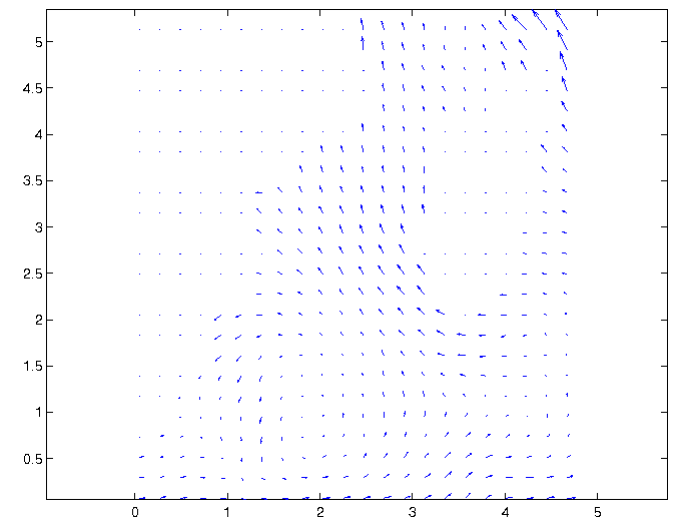

Figure 14: Plot of the given vector field $\left\{w_{i}\right\}_{i=1, \cdots, N}$.

$\mathcal{C}^{1}$ and the corresponding approximant $u_{\epsilon}^{h}$ is computed. The results are presented in Fig. 16-17. We have used a mesh refinement for the visualization: each rectangle has been subdivided into $20 \times 20$ rectangles. The relative error is equal to 0.0002522852 .
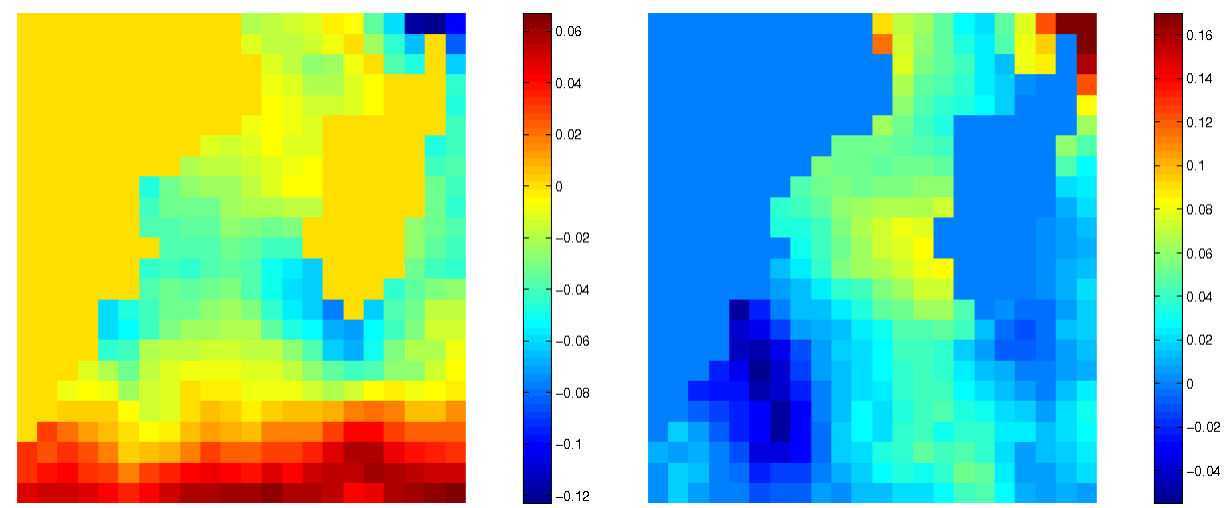

Figure 15: On the left, plot of the first component of the vector field $\left\{w_{i}\right\}_{i=1, \cdots, N}$. On the right, plot of the second component.

\section{References}

[1] L. Amodei and M.N. Benbourhim, A Vector Spline Approximation, Journal of Approximation Theory, 67(1), 51-79, 1991.

[2] D. Apprato and C. Gout, A result about scale transformation families in approximation: ap- 

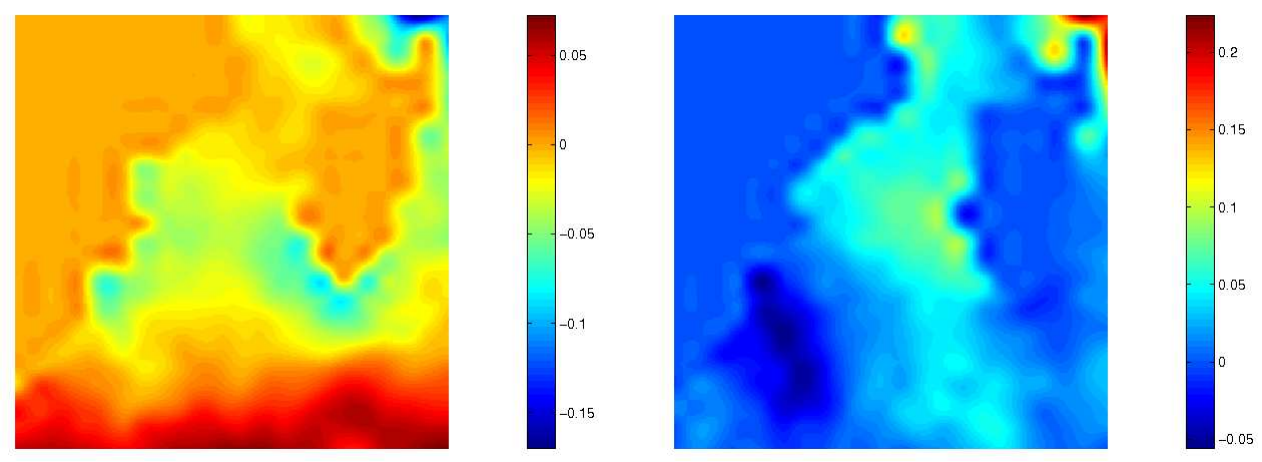

Figure 16: On the left, plot of the first component of the obtained vector field. On the right, plot of the second component.

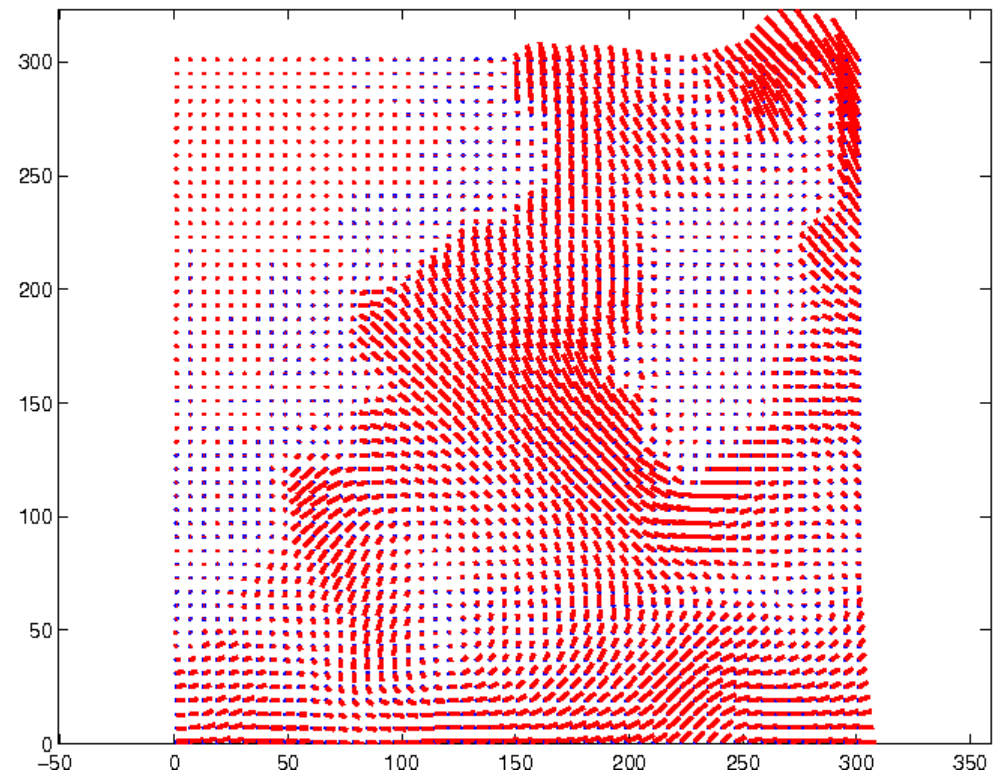

Figure 17: Plot of the obtained vector field (every six rows and columns). The blue diamond indicates the point to which the vector is attached. 
plication to surface fitting from rapidly varying data, Numerical Algorithms, 23 (2-3), 263-279, 2000.

[3] D. Apprato, C. Gout and D. Komatitsch, Surface fitting from ship track data : application to the bathymetry of the Marianas trench, Math. Geol., 34(7), 831-843, 2002.

[4] R. Arcangéli, M.C. López de Silanes and J.J. Torrens, Multidimensional minimizing splines. Theory and applications, Grenoble Sciences, Kluwer Academic Publishers, Boston, MA, 2004, xvi+261 pp., ISBN: 1-4020-7786-6.

[5] R. Arcangeli and J.L. Gout, Sur l'évaluation de l'erreur d'interpolation de Lagrange dans un ouvert de $\mathbb{R}^{n}$. Revue française d'automatique, informatique, Recherche Opérationnelle. Analyse numérique, 10 (1), pp. 5-27, 1976.

[6] G. Awanou and M. J. Lai, Trivariate spline approximations of 3D Navier-Stokes equations, Math. Comp., 74, 585-601, 2005.

[7] M.N. Benbourhim and A. Bouhamidi, Approximation of vector fields by thin plate splines with tension, Journal of Approximation Theory, 136, 198-229, 2005.

[8] M.N. Benbourhim and A. Bouhamidi, Pseudo-polyharmonic vectorial approximation for divcurl and elastic semi-norms, Numerische Mathematik, 109(3), 333-364, 2008.

[9] P.G. Ciarlet, The finite element method for elliptic problems, North Holland, Amsterdam, 1978.

[10] P. Clément, Approximation by Finite Element Functions Using Local Regularisation, RAIRO, 9ème année, R-2, 77-84, 1975.

[11] F. Dodu and C. Rabut, Vectorial interpolation using radial-basis-like functions, Comput. Math. Appl., 43(3-5), 393-411, 2002.

[12] J. Duchon, Splines minimizing rotation-invariant semi-norms in Sobolev spaces, In Constructive Theory of Functions of Several Variables, W. Schempp, K. Zeller (eds.), Lecture Notes in Mathematics, 571, 85-100, Spinger Berlin, 1977.

[13] J. Duchon, Sur l'erreur d'interpolation des fonctions de plusieurs variables par les $\mathrm{D}^{m}$ splines, RAIRO M² AN 12 (4), pp. 325-334, 1978.

[14] A.S. Dzhabrailov, Y.V. Klochkov, S.S. Marchenko and A.P. Nikolaev, The finite element approximation of vector fields in curvilinear coordinates, Russian Aeronautics (Iz VUZ), 50(2), 115-120, 2007.

[15] S. Ettl-Lowitzsch, J. Kaminski, M.C. Knauer, and G. Häusler, Shape reconstruction from gradient data, Applied Optics, 47(12), 2091-20971, 2008.

[16] C. Gout, $C^{k}$ surface reconstruction from surface patches, Computers \& Mathematics with Applications, 44(3-4), 389-406, 2002.

[17] S. Lowitzsch, Approximation and Interpolation employing divergence-free radial basis functions with applications, Dissertation, Texas A\&M University, 2002. 
[18] S. Lowitzsch, Error estimate for matrix-valued radial basis functions interpolation, J. Approx. Theory, 137(2), 238-249, 2005.

[19] M.C. López de Silanes, R. Arcangéli, Estimations de l'erreur d'approximation par splines d'interpolation et d'ajustement d'ordre $(m, s)$, (French) [Approximation error estimates for interpolating and smoothing (m,s)-splines], Numer. Math., 56(5), 449-467, 1989.

[20] M.C. López de Silanes, R. Arcangéli, Sur la convergence des $D^{m}$-splines d'ajustement pour des données exactes ou bruitées, (French) [On the convergence of fitting $D^{m}$-splines for exact or noisy data], Rev. Mat. Univ. Complut. Madrid, 4(2-3), 279-294, 1991.

[21] M.C. Lopez de Silanes, D. Apprato, Estimations de l'erreur d'approximation sur un domaine borné de $\mathrm{R}^{n}$ par $\mathrm{D}^{m}$ splines d'interpolation et d'ajustement discrètes, Numerische Mathematik 53, pp. 367-376, 1988.

[22] L. Monier, F. Brossier and F. Razafimahery, Dynamic of the currents in the Mozambique canal, Proceedings of the $30^{\text {th }}$ Jubilee International Conference on Applications of Mathematics in Engineering and Economics, 130-135, 2004.

[23] J. Nečas, Les méthodes directes en théorie des équations elliptiques, Paris, Masson, 1967.

[24] G. Strang, Approximation in the Finite Element Method, Numer. Math, 19, 81-98, 1972.

[25] Zhou, T., Han, D. and Lai, M. J., Energy Minimization Method for Scattered Data Hermite Interpolation, Journal of Applied Numerical Mathematics, vol. 58, pp. 646?659, 2008. 